\title{
Inelastic Scattering off a Nanomechanical Resonator
}

\author{
Balázs Dóra • Miklós Gulácsi
}

Received: 10 October 2008 / Accepted: 10 October 2008 / Published online: 25 October 2008

(C) The Author(s) 2008. This article is published with open access at Springerlink.com

\begin{abstract}
We study the dephasing rate of conduction electrons due to local vibrational modes. Bosonization allows us to evaluate the full $T$-matrix analytically. The inelastic scattering rate is strongly influenced by multiphonon excitations. For higher frequencies, it saturates to a finite, coupling dependent value. In the strong coupling limit, the phonon is almost completely softened, and the inelastic cross section reaches its maximal value. This represents a magnetic field insensitive contribution to the dephasing time in mesoscopic systems, in addition to magnetic impurities.
\end{abstract}

Keywords Dephasing - Inelastic scattering · Local phonon mode

PACS 73.23-b $\cdot 73.63-\mathrm{b} \cdot 72.10 . \mathrm{Fk}$

The loss of quantum coherence, occurring through inelastic processes during which some excitations are left behind and the outgoing state is not the single particle state any more, is characterized by the dephasing time, $\tau_{\phi}$. The dephasing time is reliably estimated from the low-field magnetoresistance data from the weak-localization corrections to the Drude conductivity. In general, any dynamical impurity with internal degrees of freedom can dephase conduction electrons. If the impurity changes its state, the environment felt by the electrons changes, causing dephasing.

Recently, dephasing due to magnetic impurities has been addressed successfully by relating the dephasing time to the

B. Dóra $(\bowtie) \cdot$ M. Gulácsi

Max-Planck-Institut für Physik Komplexer Systeme, Nöthnitzer

Str. 38, 01187 Dresden, Germany

e-mail: dora@pks.mpg.de inelastic cross section $[1,9,16]$. However, in many systems, Coulomb-type interaction is not the only source of correlation, which leads us to consider the interaction of electrons with local vibrational (phononic) modes. First, artificial quantum dots based on single molecules [11] (e.g. $\mathrm{C}_{60}$ ) usually distort upon the addition or removal of electrons, and can act as a quantized nanomechanical oscillator. Other realization contains suspended quantum dot cavity [13], where confined phonon modes influence the transport. Second, in many strongly correlated systems, such as heavy fermions or valence fluctuation systems, lattice vibrations are known to couple strongly to electrons. The recent discovery of magnetically robust heavy fermion behavior in filled skutterudite compound [12] $\mathrm{SmOs}_{4} \mathrm{Sb}_{12}$ renewed interest in Kondo phenomena with phononic origin [6,14].

Therefore, it is timely to investigate the source of dephasing other than magnetic impurities, such as a localized oscillator. This amounts to studying the Yu-Anderson or single impurity Holstein model given by [15]

$H=\sum_{k} \varepsilon(k) c_{k}^{+} c_{k}+g_{d} Q \Psi^{+}(\mathbf{0}) \Psi(\mathbf{0})+\frac{P^{2}}{2 m}+\frac{m \omega_{0}^{2}}{2} Q^{2}$,

which describes 3-dimensional electrons interacting with a local bosonic mode, $\Psi(\mathbf{r})$ denotes the bulk 3D electron field operator and $c_{k}$ its Fourier components [3]. This accounts for a variety of defects [3].

Due to the electron-phonon coupling $g$, the phonon mode softens as [2]

$\omega_{p \pm}=-i \Gamma \pm \sqrt{\omega_{0}^{2}-\Gamma^{2}-\Gamma \omega_{0}^{2} / \Gamma_{2}}$,

where $\Gamma_{2}=\pi \omega_{0}^{2} / 4 W \ll \omega_{0} \ll W, W$ is the bandwidth of the conduction electrons, and $\Gamma=\pi(g \rho)^{2} / 2 m$ for small $g$, 
and approaches $\Gamma_{2}$ as $g \rightarrow \infty$. Here, $\rho=1 / 2 \pi v_{F}$ is the chiral electron density of states. The explicit dependence of $\Gamma$ on $g$ cannot be determined by the bosonization approach [2]. The real part of the phonon frequency remains finite (underdamped) for $\Gamma<\Gamma_{1} \approx \Gamma_{2}\left(1-\Gamma_{2}^{2} / \omega_{0}^{2}\right)$. For $\Gamma_{1}<\Gamma<\Gamma_{2}$, the oscillatory behavior disappears from the phononic response $\left(\operatorname{Re} \omega_{p \pm}=0\right)$, and two distinct dampings characterize it (overdamped). Upon using the bosonic representation of the fermionic field [3], after lengthy calculations we determine the full many-body $s$-matrix as $[3,8]$

$s(\omega)=\left(\frac{\omega_{p+}}{\omega_{p-}}\right)^{4 \Gamma i /\left(\omega_{p+}-\omega_{p-}\right)}+F(\omega)$,

where

$F(\omega)=\int_{0}^{\infty} d t \frac{\exp (i \omega t)}{\pi t} \operatorname{Im} \exp \left[\frac{4 \Gamma\left(d\left[t \omega_{p+}\right]-d\left[t \omega_{p-}\right]\right)}{i\left(\omega_{p+}-\omega_{p-}\right)}\right]$,

$d[x]=\exp (-i x)\left(-\operatorname{Ci}(-x)+i\left(\operatorname{Si}(-x)-\frac{\pi}{2}\right)\right)$ in the underdamped case, $\operatorname{Si}(x)$ and $\operatorname{Ci}(x)$ are the sine and cosine integrals, and $\rho_{b}=k_{F}^{2} / 2 \pi^{2} v_{F}$ is the 3D bulk density of states. It contains all the non-perturbative effects of the quantum impurity introduced in (1), which stays always within the unit circle in the complex plane. The circumference of the unit circle denotes perfect elastic scattering. The total, elastic and inelastic cross section is readily evaluated from $s(\omega)$ as $[1,8,10]$

$\sigma_{\mathrm{tot}}=\frac{\sigma_{0}}{2}(1-\operatorname{Re} s(\omega))$,

$\sigma_{\text {inel }}=\frac{\sigma_{0}}{4}\left(1-|s(\omega)|^{2}\right)$,

$\sigma_{\mathrm{el}}=\frac{\sigma_{0}}{4}\left(1-2 \operatorname{Re} s(\omega)+|s(\omega)|^{2}\right)$,

where $\sigma_{0}=2 / \pi \rho_{b} v$ is the maximal value of the cross section; $\sigma_{\text {inel }}$ is directly related to the dephasing time $\tau_{\phi}$, measured in weak-localization experiments.

Figure 1 shows the total, elastic and inelastic scattering rates as a function of frequency for several $\Gamma$. At zero frequency, all rates vanish, since the electron Green function is pinned to its non-interacting value due to some generalized Fermi liquid relations [5]. For small couplings and $\omega<\operatorname{Re} \omega_{p \pm}$, the ground state contains no phonons but a filled Fermi sea, thus electrons scatter off only elastically. By exceeding this threshold $\left(\operatorname{Re} \omega_{p \pm}\right)$, the total scattering is mainly given by the inelastic cross section, which develops steps at $n \times\left|\omega_{p \pm}\right|$ (with $n$ integer), stemming from $n$ phonon excitations. Higher steps occur with smaller weight in alternating fashion. At the same time, the elastic rate exhibits a small cusp at $\omega_{0}$ due to the logarithmic singularity of the real part of electron self-energy [4].

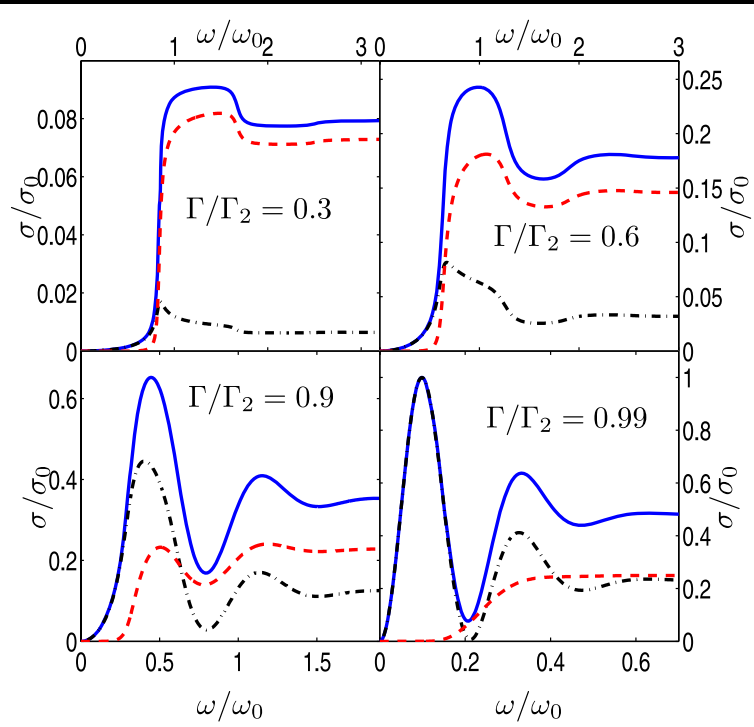

Fig. 1 (Color online) The frequency dependent scattering cross sections are shown for $W=10 \omega_{0}, \Gamma / \Gamma_{2}=0.3,0.6,0.9$ and 0.99 from left to right, top to bottom. The solid blue, red dashed and black dashed-dotted lines denote $\sigma_{\text {tot }}, \sigma_{\text {inel }}$ and $\sigma_{\mathrm{el}}$, respectively. For small frequencies, all scattering rates vanish. Then, wild oscillations show up, representing the various multiphonon ( $n$-phonon) processes, setting in at $n \times\left|\omega_{p \pm}\right|$. At higher frequencies $\left(\left|\omega_{p \pm}\right| \ll \omega \ll W\right)$, inelastic processes dominate. With increasing $\Gamma$, the weight of both elastic and inelastic processes increases. By approaching the critical coupling, the inelastic scattering rate reaches its maximal value, $\sigma_{0} / 4$, because with the vanishing phonon frequency, $\omega_{p-}$, phonon excitations become more likely, favoring inelastic scattering

For larger couplings, these sharp steps become smeared and turn into oscillations due to the finite lifetime of the phonons. The elastic scattering rate grows progressively, and becomes the dominant scattering mechanism for 1 and 2 phonon excitations. By approaching the critical coupling $\Gamma_{2}$, the inelastic scattering reaches its maximum, since the vanishing phonon energy, $\omega_{p-}$, makes arbitrary multiphonon processes possible. In two experiments on artificial quantum dots $[11,13], \omega_{0}$ fell into the $0.5-50 \mathrm{~K}$ range. These results at $T=0$ are in marked contrast to those found for Kondo impurities [1].

In conclusion, we have demonstrated through an exact solution of the single impurity Holstein or Yu-Anderson model that local vibrational modes can have a strong impact on the dephasing time of electrons. The inelastic scattering rate exhibits strong oscillations at frequencies comparable to the phonon excitation energy, and then saturates to a finite, coupling dependent value. At the extreme strong coupling limit, close to the complete softening of the phonons, the $s$-matrix vanishes and the inelastic cross section reaches its maximal value. This phonon mediated scattering mechanism is expected to be rather insensitive to the applied magnetic field, in contrast to Kondo-type impurities, and can contribute to the dephasing time in certain alloys containing dynamical defects [7, 11]. 
Acknowledgements Useful and illuminating discussions with Peter Fulde and Sergej Flach are gratefully acknowledged. This work was supported by the Hungarian Scientific Research Fund under grant number K72613.

Open Access This article is distributed under the terms of the Creative Commons Attribution Noncommercial License which permits any noncommercial use, distribution, and reproduction in any medium, provided the original author(s) and source are credited.

\section{References}

1. Borda, L., Fritz, L., Andrei, N., Zaránd, G.: Phys. Rev. B 75, 235112 (2007)

2. Dóra, B.: Phys. Rev. B 75, 245113 (2007)

3. Dóra, B., Gulácsi, M.: arXiv:0806.1680 (2008)

4. Engelsberg, S., Schrieffer, J.R.: Phys. Rev. 131, 993 (1963)

5. Hewson, A.C.: The Kondo Problem to Heavy Fermions. Cambridge University Press, Cambridge (1993)
6. Hotta, T.: J. Phys. Soc. Jpn. 76, 023705 (2007)

7. Huang, S.M., Lee, T.C., Akimoto, H., Kono, K., Lin, J.J.: Phys. Rev. Lett. 99, 046604 (2007)

8. Langreth, D.C.: Phys. Rev. 150, 516 (1966)

9. Micklitz, T., Altland, A., Costi, T.A., Rosch, A.: Phys. Rev. Lett. 96, 226601 (2006)

10. Micklitz, T., Costi, T.A., Rosch, A.: Phys. Rev. B 75, 054406 (2007)

11. Park, H., Park, J., Lim, A.K.L., Anderson, E.H., Alivisatos, A.P., McEuen, P.L.: Nature 407, 57 (2000)

12. Sanada, S., Aoki, Y., Aoki, H., Tsuchiya, A., Kikuchi, D., Sugawara, H., Sato, H.: J. Phys. Soc. Jpn. 74, 246 (2005)

13. Weig, E.M., Blick, R.H., Brandes, T., Kirschbaum, J., Wegscheider, W., Bichler, M., Kotthaus, J.P.: Phys. Rev. Lett. 92, 046804 (2004)

14. Yotsuhashi, S., Kojima, M., Kusunose, H., Miyake, K.: J. Phys. Soc. Jpn. 74, 49 (2005)

15. Yu, C.C., Anderson, P.W.: Phys. Rev. B 29, 6165 (1984)

16. Zaránd, G., Borda, L., von Delft, J., Andrei, N.: Phys. Rev. Lett. 93, 107204 (2004) 